\title{
Simulation and Characterization of Transient and Steady State Electrosprays
}

\author{
Yun Ouedraogo*1, Erion Gjonaj ${ }^{1}$, Herbert De Gersem¹ ${ }^{1}$, Christoph Steinhausen², Grazia \\ Lamanna $^{2}$, Bernhard Weigand ${ }^{2}$ \\ ${ }^{1}$ Institute for Accelerator Science and Electromagnetic Fields (TEMF), Technical University of \\ Darmstadt, Darmstadt, Germany \\ ${ }^{2}$ Institute of Aerospace Thermodynamics (ITLR), University of Stuttgart, Stuttgart, Germany \\ ${ }^{*}$ Corresponding author email: ouedraogo@temf.tu-darmstadt.de
}

\begin{abstract}
Transient electrospray atomization in the cone-jet mode is investigated by means of numerical simulations. The case of electrospray jets growing from a liquid dripping from a capillary is studied. For the characterization of the process, a particular electrospray configuration is considered for which experimental validation data are available. The droplet charge, radius and their correlations are computed for different flow rates, for transient as well as for steady state electrosprays. It is found that the charge-radius correlation of primary droplets in the initial phase of the electrospray follows a power law similar to the universal scaling law for the first atomization. However, as the electrospray develops towards steady state, the electrospray characteristics changes. The power exponent of the charge-radius correlation is still independent from the flow rate. However, this exponent deviates from that of early stage electrospray. In particular, simulations show that at steady state, the charge of primary electrospray droplets is directly proportional to their volume.
\end{abstract}

\section{Keywords}

Electrospray, Electrohydrodynamics, Finite Volume Method

\section{Introduction}

Electrospray atomization is an electrohydrodynamic process with a broad range of technical applications. Different modes of electrospraying can be observed depending on experimental parameters, such as applied voltage, electrode geometry and liquid flow rate [3, 14, 25]. Of particular interest for most applications is cone-jet electrospray. In this mode, a strong electric field applied to a conductive liquid produces a locally conical shape, from the tip of which a thin jet is ejected before atomizing into a number of microdroplets. Cone-jet electrospray is used for powder production [2], micro encapsulation [6], micro mixing [1] and nanostructured particles [26]. The technique is also used for the production of monodisperse nanoparticles [15], and as an ionization technique for mass spectrometry [7, 12]. In space propulsion applications, electrosprays are used for the production of charged droplets in efficient colloid thrusters [9, 24]. In all of these applications, a crucial property of cone-jet electrosprays is the ability to produce sprays with predictable properties such as droplet charge, droplet size and electric current $[3,23]$. Furthermore, the cone-jet mode allows to produce nearly monodisperse sprays with extremely narrow droplet size distributions $[5,11,20,21,22,23]$ for primary droplets. Therefore, extensive efforts have been made to characterize electrospray flow, in order to predict the size and charge of the atomized microdroplets from the material properties of the atomized liquids and the main experimental parameters such as flow rate and applied voltage [8, 11]. Collins [4] and later Gañán-Calvo [10] postulated universal scaling laws for the first electrospray droplet in the cone-jet mode. The validity of these laws has been established experimentally as well as by means of numerical simulations. However, it is not clear to what extent similar laws apply for the consequently emitted droplets in a transient electrospray process. In particular, of major importance for most of the applications is the characterization of the correlation between droplet charge and radius for electrosprays at steady state. 
Few experimental data are available for a systematic characterization of transient electrosprays. In [20], the electrospray atomization of a heptane based liquid dripping from a capillary with different flow rates was considered. The numerical investigation in the present paper refers to the same experimental setup as in [20]. The simulation approach, however, allows for a larger range of variation of parameters. Most importantly, the charge of electrospray droplets can be easily extracted from simulations, thus, allowing to reconstruct transient charge-radius characteristics at different stages of a developing electrospray.

The paper is constructed as follows. First, the numerical approach used for the coupled solution of the transient electroquasistatic and fluid flow problems is described. In the following, the electrospray setup, material parameters and simulation details are presented. The results obtained for a heptane electrospray are validated against experimental data taken from literature. Finally, the charge-radius correlations for the transient as well as the steady state electrosprays are presented, indicating a clear behavior corresponding to a power law scaling. These transient and steady state electrospray scalings, however, depart substantially from the charge-radius correlation predicted earlier for the first electrospray droplet.

\section{The electrohydrodynamic problem}

The simulation study is based on the coupled solution of the transient electroquasistatic and fluid flow problems. The incompressible fluid flow problem is governed by the Navier-Stokes equations,

$$
\begin{aligned}
\frac{\partial \rho \mathbf{u}}{\partial t}+\nabla \cdot \rho \mathbf{u u} & =-\nabla p+\nabla \cdot\left(\mu\left[\nabla \mathbf{u}+\nabla \mathbf{u}^{\mathrm{T}}\right]\right)+\rho \mathbf{g}+\mathbf{f}_{\mathrm{s}}+\mathbf{f}_{\mathrm{e}}, \\
\nabla \cdot \mathbf{u} & =0,
\end{aligned}
$$

where $\mathbf{u}$ denotes the fluid velocity, $\rho$ is the density, $\mu$ the dynamic viscosity and $p$ the pressure. The driving terms in (1), $f_{\mathrm{s}}$ and $\mathrm{f}_{\mathrm{e}}$, denote the surface tension force density acting at the interface between the different fluids and the electric force density, respectively. The former is derived from a surface force density $\hat{\mathbf{f}}_{\mathrm{s}}$, itself related to the phase boundary properties according to Young-Laplace equation,

$$
\hat{\mathbf{f}}_{\mathrm{s}}=2 \gamma H \mathbf{n},
$$

where $\gamma$ is the surface tension characterizing the fluid-fluid interface, $H$ the interface mean curvature and $\mathbf{n}$ the normal.

The electric field $\mathbf{E}$ in the fluid is derived from the gradient of an electric potential, $\mathbf{E}=-\nabla \Phi$. We assume that the diffusion of free charge carriers is negligible compared to electric field induced motion [10]. Under this assumption, the conservation equation for the free charge density, $\varrho_{\mathrm{e}}$, in the fluid can be written as

$$
\frac{\partial \varrho_{\mathrm{e}}}{\partial t}+\nabla \cdot\left(\varrho_{\mathrm{e}} \mathbf{u}\right)=\nabla \cdot \kappa \nabla \Phi,
$$

where $\varepsilon$ and $\kappa$ are, respectively, the permittivity and ohmic conductivity of the media. Hereby, the electric potential distribution is related to the free charge distribution by Gauss's law,

$$
\nabla \cdot \varepsilon \nabla \Phi=-\varrho_{\mathrm{e}} \cdot
$$

Finally, given the electric field distribution, the electric force density applied on the fluid can be obtained from the Maxwell stress tensor as

$$
\mathbf{f}_{\mathrm{e}}=\nabla \cdot\left(\varepsilon \mathbf{E} \otimes \mathbf{E}-\frac{1}{2} \varepsilon E^{2} \mathbf{I}\right)
$$

Equations (1)-(6) describe the coupled electroquasistatic-hydrodynamic electrospray problem. For the numerical solution of this problem, the Finite Volume Method is used, with a Volume of 
Fluid formulation for the representation of the liquid-gas interface [13]. This notably allows to express the surface tension force density $\hat{f}_{\mathrm{s}}$ as a body force $\mathrm{f}_{\mathrm{s}}$ acting at the fluid-fluid interface. Due to the rotational invariance of the cone-jet geometry, the electrosprays are simulated assuming axisymmetry of the problem. The implementation used for all electrospray simulations presented in the paper is based on the OpenFOAM library. This implementation is thoroughly described in [16] and will, therefore, not be further discussed in this paper. It should be noted, however, that this implementation includes the application of a parallel adaptive mesh refinement approach with dynamic load balancing as described in [18]. Such an approach is required to achieve the necessary level of numerical resolution for the small electrospray droplets, while keeping computational costs acceptable.

\section{Numerical setup}

The simulated setup is shown in Figure 1. It consists essentially of a capillary that provides a dripping liquid with variable flow rates. The grounded metallic tip of the capillary and a plane electrode located at a distance of a few centimeters below the capillary form the electrostatic gap that is necessary for electrospray atomization. A voltage of a few $\mathrm{kV}$ is applied at the electrode. Since the fluid is continuously filled in, a steady cone-jet electrospray is eventually obtained in a proper range of mass flow rate and applied voltage [23]. The boundary conditions used in the computation are detailed in Table 1.

In the following, simulation results for electrospray atomization of heptane $+0.3 \%$ stadis 450 with $U_{0}=4 \mathrm{kV}$ and $\varphi \in\left[0.39 \mathrm{ml} \mathrm{h}^{-1}, 28 \mathrm{ml} \mathrm{h}^{-1}\right]$ are presented. The simulations were run with a maximal level of mesh refinement of $l_{\max }=5$ for flow rates $\varphi \leq 12 \mathrm{ml} \mathrm{h}^{-1}$ and $l_{\max }=4$ for $\varphi>12 \mathrm{ml} \mathrm{h}^{-1}$, with a resolution of $\Delta \rho=\Delta z=15 \mu \mathrm{m}$ at the base level (unrefined mesh).

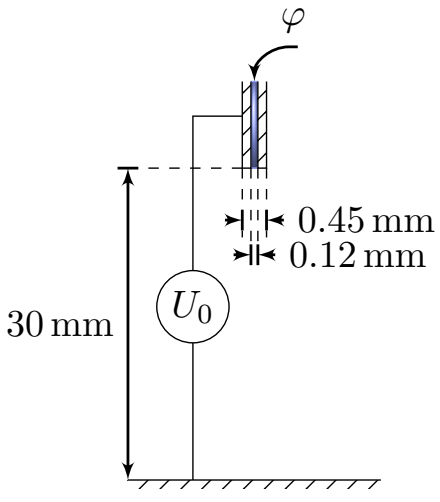

(a)

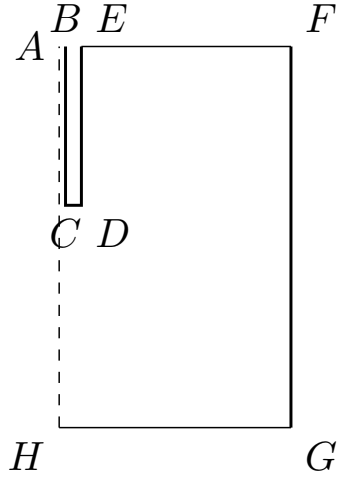

(b)

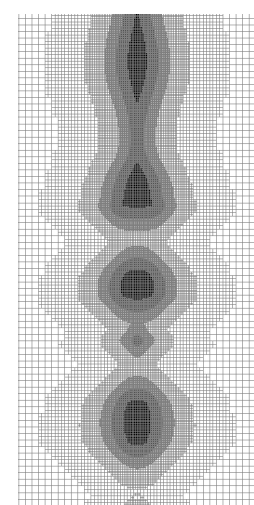

(c)

Figure 1. (a) Schematic view and main parameters of the simulation domain. (b) Labels of the boundary conditions applied to the computational domain (see Table 1). (c) Example view of mesh adaption for typical electrospray droplets.

\begin{tabular}{|c|c|c|c|c|c|}
\hline \multirow[t]{2}{*}{ Boundary } & \multicolumn{5}{|c|}{ Variable } \\
\hline & $p_{\mathrm{d}}$ & $\mathrm{u}$ & $\alpha$ & $\Phi$ & $\varrho_{\mathrm{e}}$ \\
\hline $\begin{array}{l}\text { Inlet } \\
(\mathrm{AB})\end{array}$ & $\nabla_{n} p_{\mathrm{d}}=0$ & $\mathbf{u} \cdot \mathbf{n}=\frac{\varphi}{\pi r_{\text {inlet }}^{2}}$ & $\alpha=1$ & $\nabla_{n} \Phi=0$ & $\nabla_{n} \varrho_{\mathrm{e}}=0$ \\
\hline $\begin{array}{l}\text { Capillary surface } \\
\text { (BCDE) }\end{array}$ & $\nabla_{n} p_{\mathrm{d}}=0$ & $\mathbf{u}=0$ & $\nabla_{n} \alpha=0$ & $\Phi=0$ & $\nabla_{n} \varrho_{\mathrm{e}}=0$ \\
\hline $\begin{array}{l}\text { Free stream surface } \\
\qquad(E F G)\end{array}$ & $p_{0}-\frac{1}{2} \rho|\mathbf{u}|^{2}$ & $\nabla_{n} \mathbf{u}=0$ & $\begin{array}{c}\nabla_{n} \alpha=0 \text { if } \mathbf{u} \cdot \mathbf{n} \leq 0 \\
0 \text { otherwise }\end{array}$ & $\nabla_{n} \Phi=0$ & $\nabla_{n} \varrho_{\mathrm{e}}=0$ \\
\hline $\begin{array}{l}\text { Electrode surface } \\
(\mathrm{GH})\end{array}$ & $\nabla_{n} p_{\mathrm{d}}=0$ & $\mathbf{u}=0$ & $\nabla_{n} \alpha=0$ & $\Phi=U_{0}$ & $\nabla_{n} \varrho_{\mathrm{e}}=0$ \\
\hline
\end{tabular}

Table 1. Boundary conditions in the simulations.

Note that the above model as well as the applied parameters correspond exactly to the experi- 
mental setup described in [23]. This choice is intentional. It allows for a direct validation of the numerical results against the reported experimental data as described below.

\section{Results and discussion}

The cone-jet with subsequent atomization occurs nearly immediately after the liquid starts dripping from the capillary. Figure 2 depicts a typical electrospray situation obtained for an applied voltage of $4 \mathrm{kV}$ and a liquid flow rate of $6.2 \mathrm{ml} \mathrm{h}^{-1}$. As seen in the figure, along with the primary electrospray droplets, satellite ejections of much smaller size (and charge) may occur.
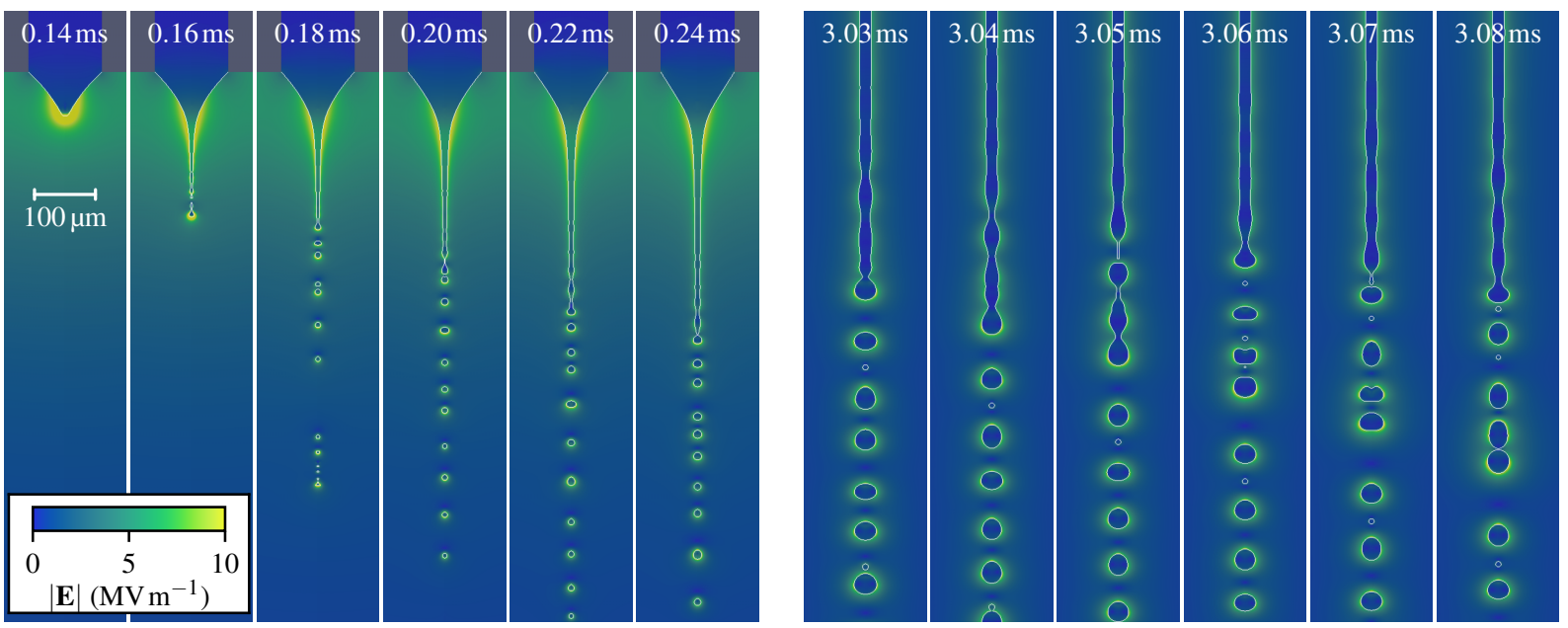

Figure 2. Onset and dynamics of electrospray in the cone-jet mode including primary and satellite droplets.

The mean size of primary droplets increases over time, until steady state is reached. This behavior is illustrated in Figure 3, where the droplet size is depicted vs. the time of their ejection for different flow rates. Steady state is typically reached within a few milliseconds, after several hundreds of primary droplets have been ejected. This figure, however, depends strongly on the flow rate since the transition to steady state is much faster for smaller flow rates.

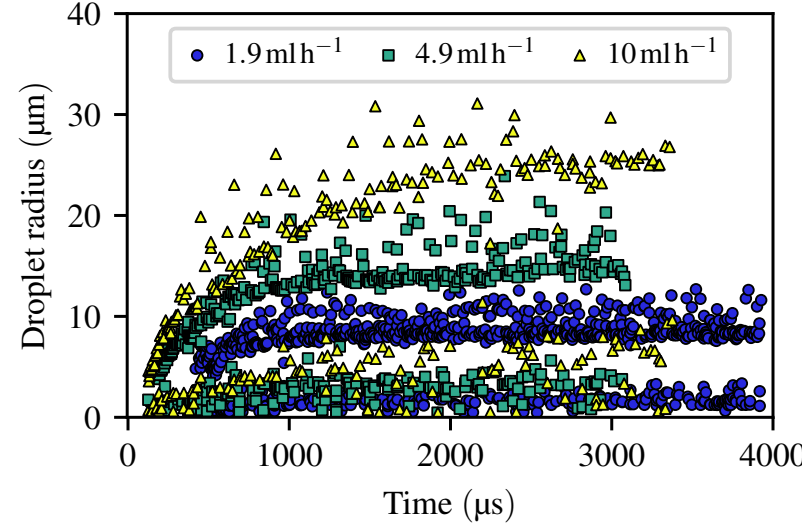

(a)

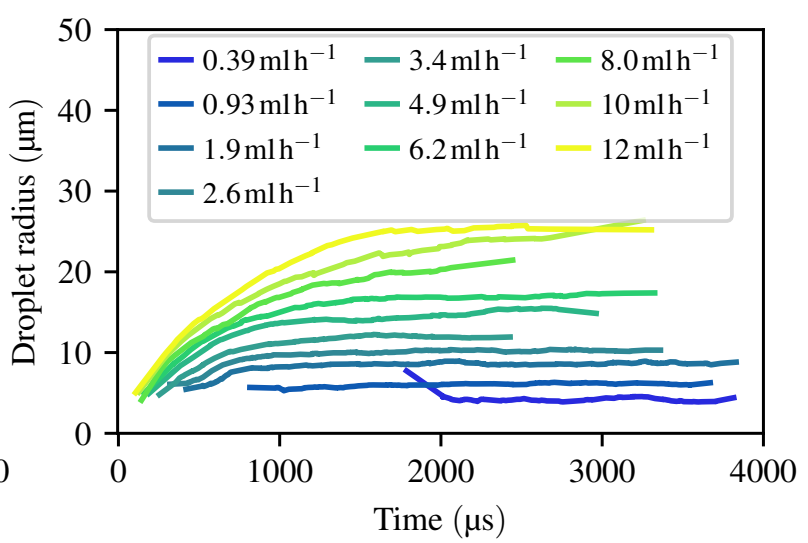

(b)

Figure 3. (a) Individual droplet sizes as a function of emission time for different flow rates. Note that for each of the electrosprays, primary and satellite droplets can be clearly distinguished by size. (b) Average size of primary droplets for the simulated flow rates.

In order to validate simulations, the computed droplet sizes at steady state for different flow rates are compared with the experimental results reported in [23]. A mean droplet size is evaluated for each flow rate from the last 50 ejections after steady state has been reached. The comparison depicted in Figure 4 shows that simulation results are in good agreement with the experiments, with a maximum relative deviation of $\sim 20 \%$. 


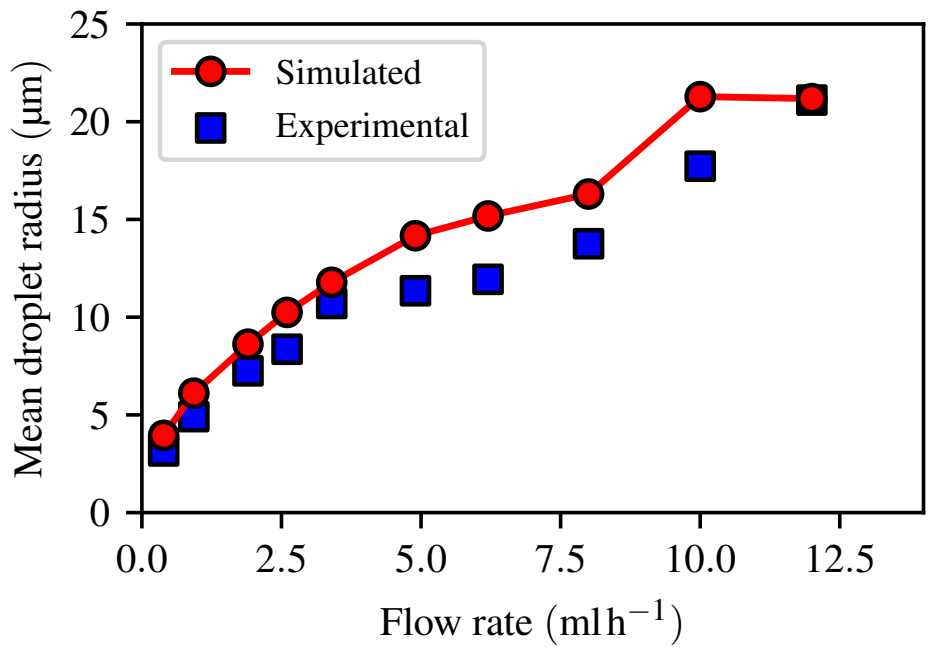

Figure 4. Comparison of simulated mean droplet radius with experimental results from [23], Figure 2(a).

The other quantity of interest that is not easily accessible experimentally is droplet charge. More specifically, one is interested in the relationship between the charge and size of electrospray droplets. Scaling laws relating droplet charge to its size have been proposed, in particular, for the very first ejected droplet $[10,4,17]$. Relations of the type $q \propto r^{\alpha}$ are suggested, where $q$ denotes the net charge of the droplet, $r$ its radius and $\alpha$ is a scaling exponent, typically, in the range between 1.5 and 3 .

In the following, we look for similar scaling laws that would however apply to the different stages of electrospray rather than just to the first ejected droplet. Figure 5 depicts a scatter plot of the charges and radii of all droplets obtained by electrospray atomization until steady state is reached for three different flow rates. Primary and satellite droplets are clearly seen to obey different scaling laws. Independently from the flow rate, the charge-radius characteristics for the primary ejections scales as $q \propto r^{1.81}$. However, the charge of satellite droplets scales as $q \propto r^{3}$ for each electrospray, with a proportionality constant decreasing with increasing flow rate.

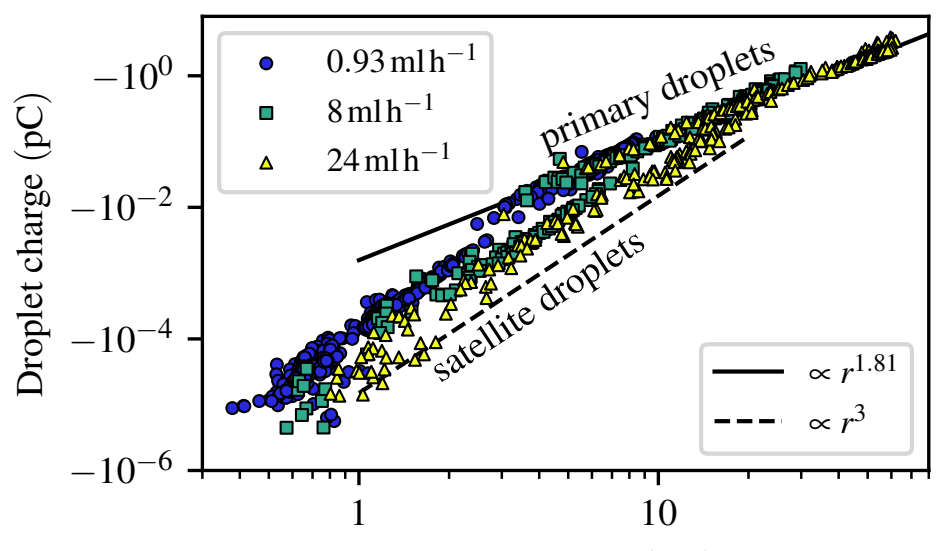

Droplet radius $(\mu \mathrm{m})$

Figure 5. Charge-radius characteristics for different flow rates. Primary ejections scale as $q \propto r^{1.81}$ over the range of considered flow rates. For clarity, data corresponding to only a few flow rates is shown.

At steady state, due to instabilities in the jet and perturbations originating from prior ejections, the size of ejected droplets varies slightly. The distribution of primary droplet sizes and net charge for a few different flow rates at steady state are shown in Figure 6a. While the size 
distribution is relatively narrow, the associated charge distribution is significantly broader. This behavior was observed experimentally in [5], where it was concluded to be indicative of a scaling of the form $q \propto r^{3}$ between the size and charge of primary droplets.

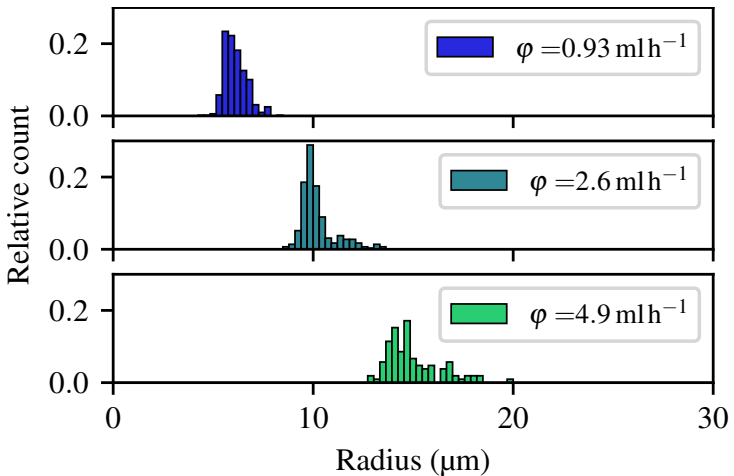

(a)

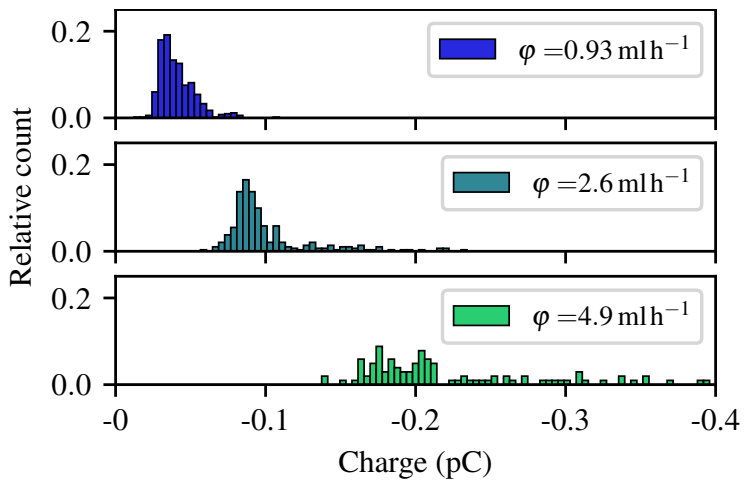

(b)

Figure 6. (a) Distribution of primary droplet sizes at steady state for different flow rates. (b) Distribution of primary droplet charge at steady state for different flow rates.

In order to investigate this relation, the charge and radius of primary droplets at steady state are shown in Figure 7a. It is clear that, once steady state is reached, the net charge of primary droplets scales as their individual volume. This is however not true for the earlier stages of the electrospray, as shown in Figure 7b. Initially, a lower exponent for the power law is observed, before increasing to reach the steady state value. This indicates that the droplet charging dynamics shortly after electrospray onset might be substantially different than for a steady state electrospray.

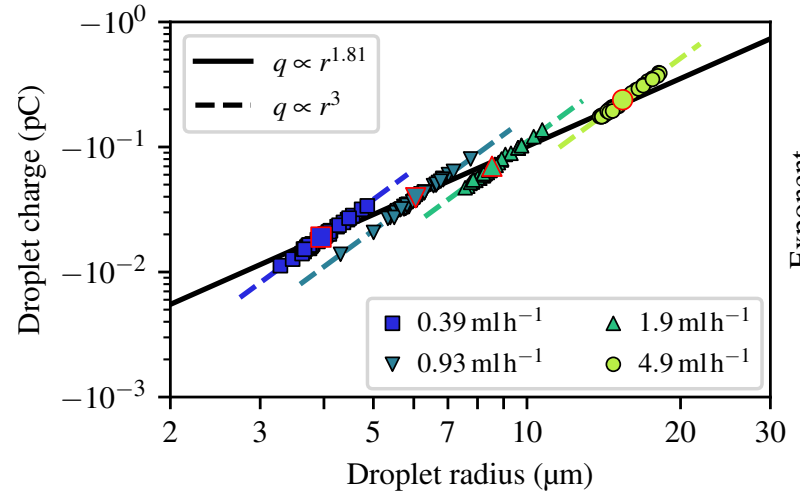

(a)

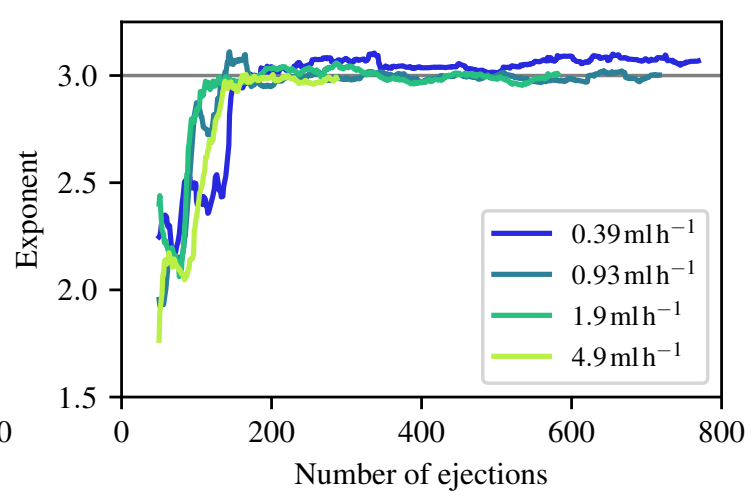

(b)

Figure 7. (a) Charge and size of the last 50 primary ejections for different flow rates. For each flow rate, the corresponding average charge and radius is indicated with a larger, red-framed marker. The curve $q \propto r^{1.81}$ shown in Figure 5 is reproduced as a reference. (b) Exponent of the power law as a function of time. 50 consecutive primary droplets are used for the fitting at each data point.

The similar observation for the charge-radius scaling at steady state was done in [5]. This behavior was explained by the Rayleigh's capillary breakup theory for low viscosity cylindrical jets. Under the assumptions that the charge at the surface of the jet does not significantly impact the breakup dynamics and the charge of the jet is frozen at the surface of the liquid during the breakup process, a steady state scaling as $q \propto r^{3}$ was predicted.

Following this argument, the ratio between the radius of ejected primary droplets and the radius of the jet should approach 1.89, as derived from Rayleigh's theory. Figure 8b shows the ratio of the average primary droplet radius to the average jet radius for the electrosprays at different flow rates. The calculated ratios are close to the curve of slope 1.89, so that the results 
are consistent with the observed correlation between the charge and radius of the atomized droplets. Note that the profile of the jets deviates slightly from the cylindrical shape assumed by the theory, as shown in Figure 8a. The small systematic deviation from the theoretical curve of slope 1.89 can be attributed to this effect.

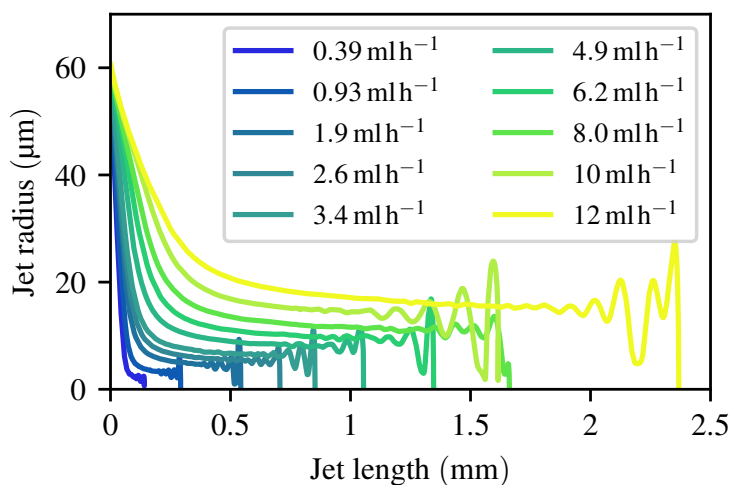

(a)

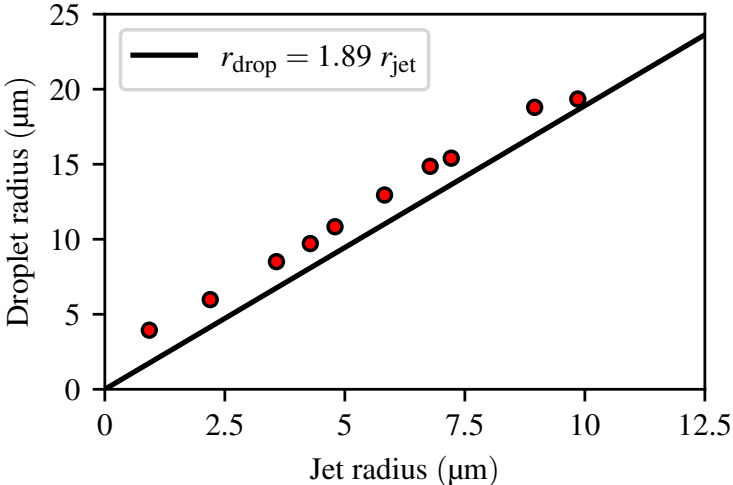

(b)

Figure 8. (a) Profile of the jet at the last simulated time step (steady state) for different flow rates. (b) Ratio between the average primary droplet radius and jet radius for the different jets.

\section{Conclusions}

A VoF-based simulation approach is applied for the investigation of droplet and charge dynamics in electrospray atomization. The charge and size of individual ejections can be extracted from numerical simulations to characterize electrosprays, both during the initial transients and at steady state. The primary droplets of the characterized electrosprays exhibit charge-radius correlations in the form of power laws with various exponents. The charge-radius correlation of the primary droplets seem to follow a unique power law that is independent from the applied flow rate. However, the correlations for individual electrosprays are found to differ significantly between the early stage and steady state of the electrospray. At steady state, the correlations for each individual electrosprays indicate that the charge of primary droplets is directly proportional to their volume.

\section{Acknowledgements}

The authors gratefully acknowledge the German Research Foundation (DFG) for the financial support of the project SFB-TRR 75 under the project number 84292822.

\section{Nomenclature}

$\begin{array}{ll}\gamma & \text { Surface tension }\left[\mathrm{N} \mathrm{m}^{-1}\right] \\ \varepsilon & \text { Permittivity }\left[\mathrm{F} \mathrm{m}^{-1}\right] \\ \kappa & \text { Electrical conductivity }\left[\mathrm{S} \mathrm{m}^{-1}\right] \\ \mu & \text { Dynamic viscosity }[\mathrm{Pa} \mathrm{s}] \\ \rho & \text { Density }\left[\mathrm{kg} \mathrm{m}^{-3}\right] \\ \varrho_{\mathrm{e}} & \text { Charge density }\left[\mathrm{C} \mathrm{m}^{-3}\right] \\ \varphi & \text { Flow rate }\left[\mathrm{m}^{3} \mathrm{~s}^{-1}\right] \\ \Phi & \text { Electric potential }[\mathrm{V}] \\ \mathbf{E} & \text { Electric field }\left[\mathrm{V} \mathrm{\textrm {m } ^ { - 1 } ]}\right. \\ \mathbf{f}_{\mathrm{s}} & \text { Surface tension force density }\left[\mathrm{N} \mathrm{m}^{-3}\right] \\ \mathbf{f}_{\mathrm{e}} & \text { Electric force density }\left[\mathrm{N} \mathrm{m}^{-3}\right] \\ \mathbf{g} & \text { Gravitational acceleration }\left[\mathrm{m} \mathrm{s}^{-2}\right] \\ H & \text { Interface mean curvature }\left[\mathrm{m}^{-1}\right]\end{array}$


n Interface normal

$p \quad$ Pressure $[\mathrm{Pa}]$

$p_{\mathrm{d}} \quad$ Dynamic pressure $[\mathrm{Pa}]$

$q \quad$ Droplet charge [C]

$r \quad$ Droplet radius [m]

u Velocity $\left[\mathrm{m} \mathrm{s}^{-1}\right.$ ]

\section{References}

[1] Borra, J.-P., Camelot, D., Chou, K.-L., Kooyman, P. J., Marijnissen, J. C. M., Scarlett, B., 1999, Journal of Aerosol Science, 30 (7), pp. 945-958.

[2] Borra, J.-P., Camelot, D., Marijnissen, J. C. M., Scarlett, B., 1997, Journal of Electrostatics, 40-41, pp. 633-638.

[3] Cloupeau, M., Prunet-Foch, B., 1994, Journal of Aerosol Science, 25 (6), pp. 1021-1036.

[4] Collins,R. T., Sambath, K., Harris M. T., Basaran, O. A., 2013, Proceedings of the National Academy of Sciences, 110 (13), pp. 4905-4910.

[5] de Juan, L., Fernández de la Mora, J., 1997, Journal of Colloid and Interface Science, 186 (2), pp. 280-293,

[6] Enayati, M., Chang, M.-W., Bragman, F., Edirisinghe, M., Stride, E., 2011, Colloids and Surfaces A: Physicochemical and Engineering Aspects, 382 (1), pp. 154-164.

[7] Fenn, J. B., Mann, M., Meng, C. K., Wong, S. F., Whitehouse, C. M., 1989, Science, 246 (4926), pp. 64-71.

[8] Fernández de la Mora, J., Loscertales, I. G., 1994, Journal of Fluid Mechanics, 260, pp. 155-184.

[9] Gamero-Castaño, M., Hruby. V., 2001, Journal of Propulsion and Power, 17 (5), pp. 977987.

[10] Gañán-Calvo, A. M., López-Herrera, J. M., Rebollo-Muñoz, N., Montanero. J. M., 2016, Scientific Reports, 6:32357,

[11] Gañán-Calvo, A. M., Dávila, J., Barrero. A., 1997, Journal of Aerosol Science, 28 (2), pp. 249-275.

[12] Gaskell., S. J., 1997, Journal of Mass Spectrometry, 32 (7), pp. 677-688.

[13] Hirt, C. W., Nichols., B. D., 1981, Journal of Computational Physics, 39 (1), pp. 201-225.

[14] Jaworek, A., Krupa, A., 1999, Journal of Aerosol Science, 30 (7), pp. 873-893.

[15] Lenggoro, I. W., Okuyama, K., Fernández de la Mora, J., Tohge, N., 2000, Journal of Aerosol Science, 31 (1), pp. 121-136.

[16] Ouedraogo, Y., Gjonaj, E., Weiland, T., De Gersem, H., Steinhausen, C., Lamanna, G., Weigand, B., Preusche, A., Dreizler, A., Schremb, M., 2017, International Journal of Heat and Fluid Flow, 64, pp. 120-128.

[17] Pillai, R., Berry, J., Harvie, D., Davidson, M. , 2016, Soft Matter, 12.

[18] Rettenmaier, D., Deising, D., Ouedraogo, Y., Gjonaj, E., De Gersem, H., Bothe, D., Tropea, C., Marschall. H., 2019, SoftwareX, 10:100317.

[19] Saville, D. A., 1997, Annual Review of Fluid Mechanics, 29 (1), pp. 27-64.

[20] Tang K., Gomez, A., 1994, Journal of Aerosol Science, 25 (6), pp. 1237-1249.

[21] Tang K., Gomez, A., 1994, Physics of Fluids, 6 (7), pp. 2317-2332.

[22] Tang K., Gomez, A., 1995, Journal of Colloid and Interface Science, 175 (2), pp. 326-332.

[23] Tang K., Gomez, A., 1996, Journal of Colloid and Interface Science, 184 (2), pp. 500-511.

[24] Velásquez-García, L. F., Akinwande, A. I., Martínez-Sánchez, M., 2006, Journal of Microelectromechanical Systems, 15 (5), pp. 1272-1280.

[25] Verdoold, S., Agostinho, L. L. F., Yurteri, C. U., Marijnissen, J. C. M., 2014, Journal of Aerosol Science, 67, pp. 87-103.

[26] Yurteri, C. U., Hartman, R. P. A., Marijnissen, J. C. M., 2010, KONA Powder and Particle Journal, 28, pp. 91-115. 